\title{
Spheroid-Based Approach to Assess Tissue Relevance of Analysis of Dispersed-Settled Tissue Cells by Cytometry of Reaction Rate Constant
}

\author{
Vasilij Koshkin, Mariana Bleker de Oliveira, Chun Peng, Laurie E. Ailles, Geoffrey Liu, Allan Covens, and
} Sergey N. Krylov*

Table of Contents

Section

Page

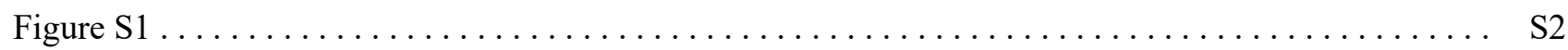

Note S1: Extraction of kinetic traces from spheroid ZT stacks $\ldots \ldots \ldots \ldots \ldots \ldots \ldots \ldots \ldots \ldots \ldots$

\section{Other Supporting Files}

File Name

ImageStacks.zip

KineticTraces.zip

KineticHistograms.zip

\section{Description}

T image stacks of monolayer cells (T stacks 1,2) and dispersed-settled spheroidal cells (T stacks 3,4) as well as ZT image stacks of intact spheroids (ZT stacks 1-6) which were used for extraction of kinetic traces utilized an subsequently building kinetic cytometry histograms. Different T stacks and different ZT stack correspond to experiments performed on different days with new samples.

Each T stack contains 60 images spaced 3 min in time; recording one image took $1.69 \mathrm{~s}$. ZT stacks contain different numbers of images as spheroids varied in size and required different numbers of horizontal sections with different $Z$ positions for every time point. Accordingly ZT stacks 1-6 contain for every time point $8,10,7,6,9$, and 5 images respectively. The numbers of time points in ZT stacks $1-6$ are 13, 14, 8, 10, 10, and 10, respectively. Totals numbers of images in ZT stacks $1-6$ are 104, 140, 56, 60, 90, and 50 , respectively. Completion of single $Z$ stack took approximately $10 \mathrm{~s}$ and one $Z$ stack was done every $3 \mathrm{~min}$. The start of every next cycle of images with varying $Z$ can be identified by taking into account the number of images in a $Z$ stack. For example, for ZT stack 1, a $Z$ stack for every time point contain 8 images, accordingly the first $Z$ stack will start with image 1 , the second $Z$ stack will start with image 9 and so on. Similarly, for ZT stack 2, a Z stack for every time point contain 10 images, accordingly the first $Z$ stack will start with image 1 , the second $Z$ stack will start with image 11 and so on.

Kinetic traces of individual spheroid cells, dispersed-settled spheroidal cells and monolayer cells obtained from time dependence of fluorescence intensity calculated from the image stacks. Column $\mathrm{A}(\mathrm{X})$ shows image number in a sequence of consecutive images separated by $3 \mathrm{~min}$; accordingly, this column can be considered as time if the number is multiplied by $3 \mathrm{~min}$. Each column to the right of column $\mathrm{A}(\mathrm{X})$ contains data on time dependence of relative fluorescence intensity from a single cell.

Kinetic cytometry histograms: $k_{\mathrm{MDR}}$ distributions in populations of outer cells in intact spheroids, dispersed-settled spheroidal cells, and monolayer cells. The number of cells in each histogram is 347 . 
a

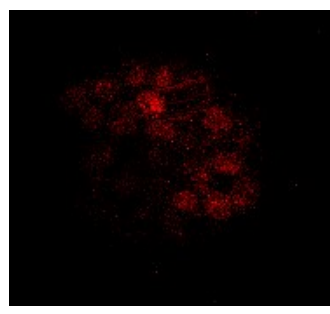

b

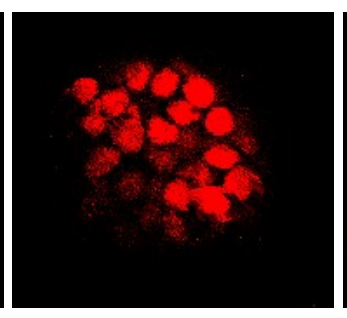

C

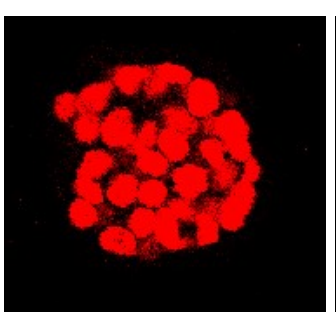

d

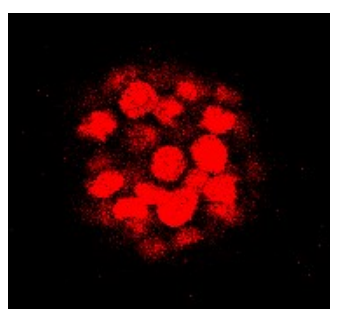

e

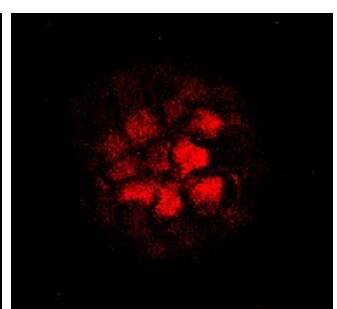

Figure S1. Sequential fluorescence images of a multicellular spheroid (A2780 ovarian cancer cells) in horizontal planes spaced vertically. The distance between the adjacent planes was $15 \mu \mathrm{m}$. Images were taken from the top to the bottom of the spheroid: $a-b)$ images above the equatorial plane; $c$ ) image in the equatorial plane; $d-$

e) images below the equatorial plane. Cells' boundaries were identified with propidium iodide staining.

\section{Note S1: Extraction of kinetic traces from spheroid ZT stacks}

Spheroid ZT stacks (archived in ZTseries.zip), were evaluated as specified below:

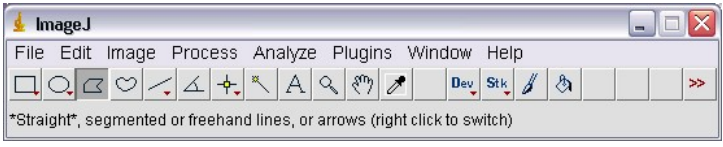

*Straight*, segmented or freehand lines, or arrows (right click to switch)
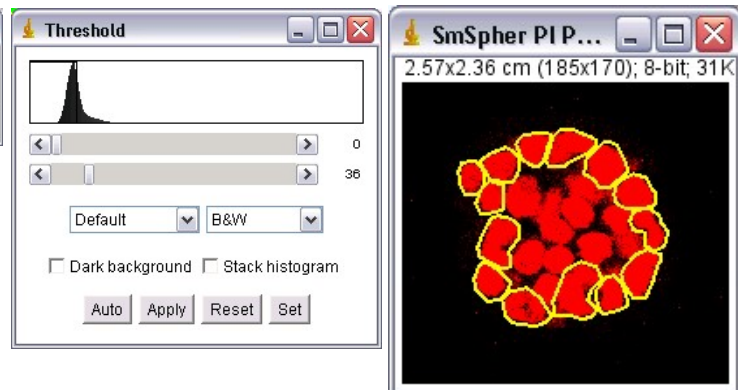

1. To facilitate cell identification, $\mathrm{Z}$ stacks of spheroids treated with PI/saponin (after the kinetic MDR assay) were open in ImageJ. Thresholds were adjusted for matching manual cell identification ("gold standard") and applied to individual $\mathrm{Z}$ sections (a typical equatorial section is shown). Oouter cell contours were selected and saved as ROIs (regions of interest) in ROI Manager.
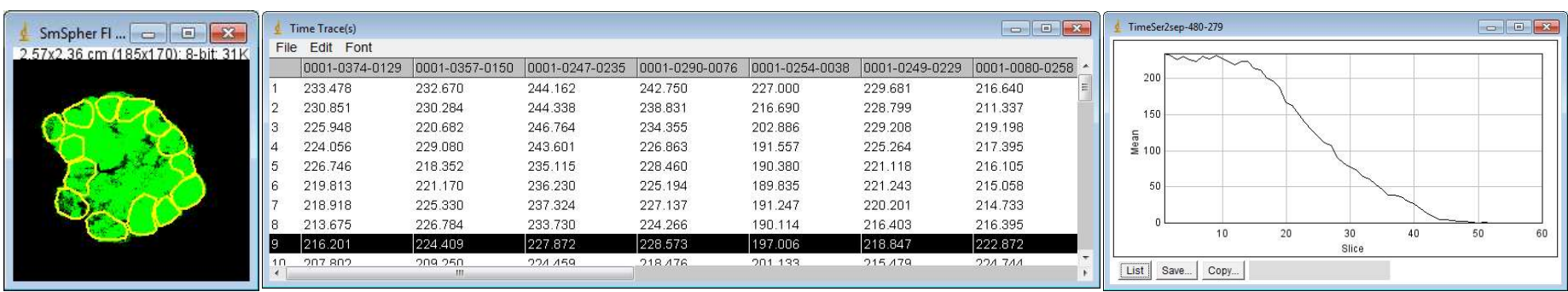

2. ROIs determined in Step 1 were applied to ZT stacks acquired in the process of kinetic MDR assay. Time Series Analyser plugin was used to form time lapse ( $T$ ) sequence of images for each $Z$ section. Analysis of these sequences produced individual cell (ROI) kinetics in a tabular form and averaged cell population kinetics in a graphical form. 